\section{Ingeniería}

CONTENIDO

Artículos

Análisis numérico de un sistema de aire acondicionado empleando
mallas estructuradas y no estructurades A

Funciones de regresión para caudales extremos en la Vertiente Rojas, Nazareth; Aguilar, José Francisco; Solís, Hernán. Evaluación de daños por agresión ambiental en viviendas
de concreto reforzado. Solís, Rómel; Moreno, Eric; Jiménez, Felipe; Rosas, Victorino.

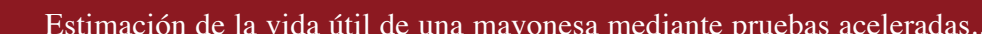
Estimación de la vida útil de una mayonesa
García, Claudia; Molina, Manuel.

Inestabilidad oscilatoria de tensión debido a los motores de inducción …….....................65-75 rde, Gustavo; Araya, Eddie.

Estabilidad y colapso de tensión en sistemas eléctricos Araya, Eddie.

Obtención de pulpa a la soda antraquinona de Tectona grandis creciendo en Costa Rica...........................

Protocolo evaluador para el manejo de desechos y la seguridad ocupacional
aplicado al laboratorio clínico del Hospital San Francisco de Asís de Grecia. Ruiz, Francisco.

9. Euler: su contex
Vargas, Celso.

10. Reserva de resistencia de edificios porticados de concreto armado disenaados conforme al ACI-318/ IBC 2006 ........ Análisis y comentarios

Herrera, Rodolfo.

breve semblanza.

Trabajos de Graduación 2007

Lista de proyectos de graduación de grado y posgrado

Normas

$\sum_{\text {EDTORAL }}$

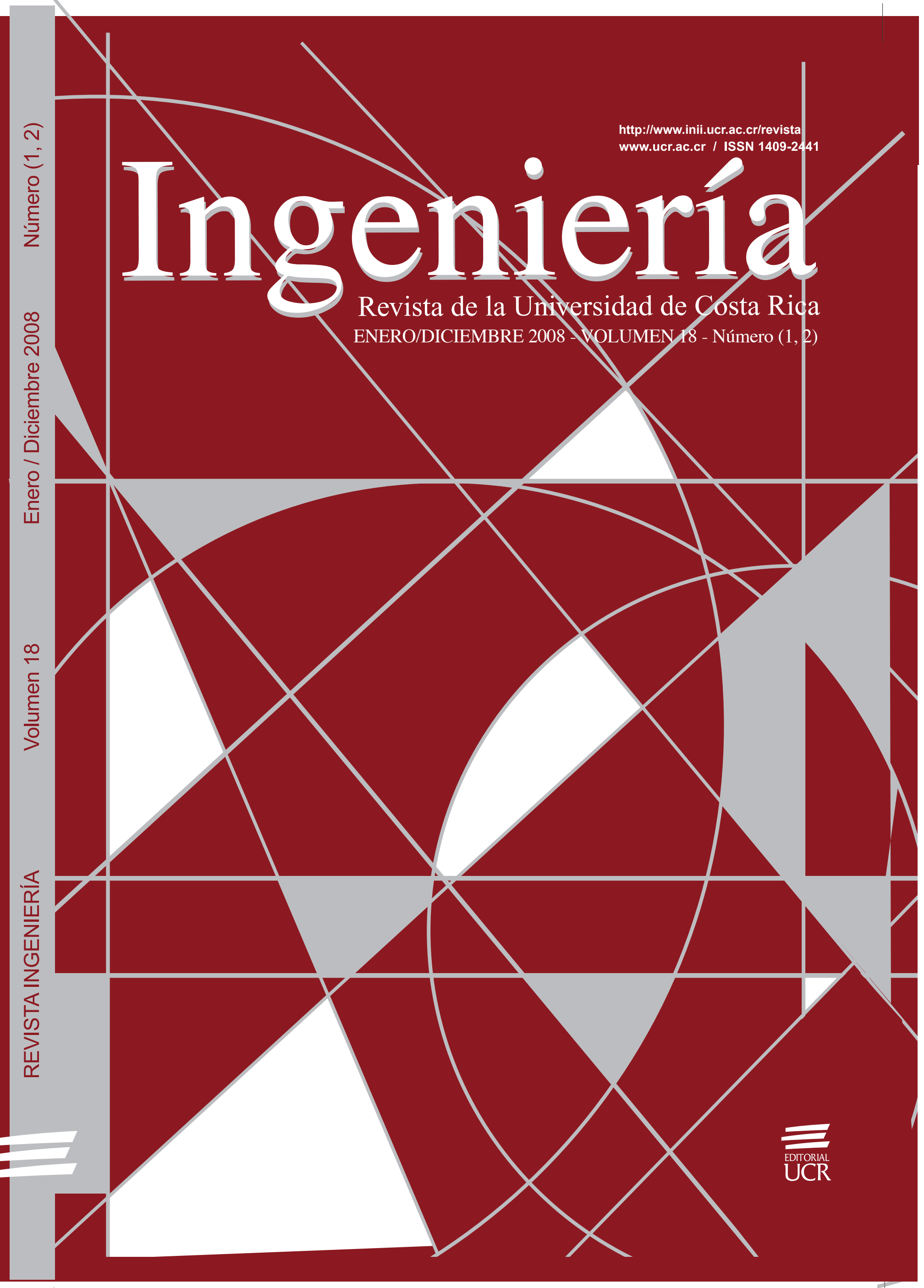




\title{
EVALUACIÓN DE DAÑOS POR AGRESIÓN AMBIENTAL EN VIVIENDAS DE CONCRETO REFORZADO
}

\author{
Rómel Solís Carcaño \\ Eric Moreno \\ Felipe Jiménez Torres \\ Victorino Rosas Pérez
}

\section{Resumen}

El concreto reforzado es un material muy eficaz para soportar esfuerzos mecánicos, pero puede resultar vulnerable cuando es sometido a ambientes severos, como es el caso de las costas marinas. Procesos electroquímicos que tienen lugar en el acero de refuerzo, y reacciones químicas entre los productos hidratados del cemento y los compuestos que penetran y se difunden en el concreto, producen deterioros en las construcciones; daños que afectan su durabilidad y reducen la seguridad y calidad de servicio. En el presente escrito se reporta la evaluación de una unidad habitacional situada en la ciudad costera de Chetumal, en el sureste de México, desde el punto de vista de su durabilidad ante los agentes de medio ambiente. En una muestra de viviendas construidas con muros de concreto reforzado se evaluó la calidad del concreto y se realizaron pruebas electroquímicas al acero de refuerzo; también se midieron las concentraciones de cloruros y sulfatos en el concreto, y se estimó la pérdida de alcalinidad del concreto. Los resultaron mostraron que el concreto utilizado fue de una calidad inferior a la necesaria y una probabilidad media de que esté ocurriendo la corrosión en el acero de refuerzo a la edad de quince años. Se concluye con el pronóstico de que, en un lapso de diez años, el proceso corrosivo del acero debe haberse generalizado y deben presentarse daños notorios en las viviendas.

Palabras clave: corrosión, carbonatación, viviendas de interés social, cargas ambientales.

\begin{abstract}
Reinforced concrete is a very effective material to support mechanical efforts, but it can be vulnerable when it is placed under severe atmospheres, as it is the case in the marine coasts. Electrochemical processes that take place in the reinforcing steel, and chemical reactions between hydrated cement products and the compounds which penetrate and spread into the concrete, produce deteriorations in the constructions; damages that affect their durability and reduce the security and quality service. This paper reports the evaluation of a housing development located in the coastal city of Chetumal, in Southeastern Mexico, from the point of view of its durability against the ambient agents. In a sample of houses constructed with reinforced concrete walls the concrete quality was evaluated and electrochemical tests were made to the steel; also, concentrations of chlorides and sulphates in the concrete were measured, and the loss of alkalinity of the concrete was valued. The result exposed that the concrete has inferior quality than the necessary and middle probability that it is happening the corrosion in the steel of reinforcement at the age of 15 years. It is concluded with the prognosis than in ten years, the corrosion process of the steel will be generalized and damages in all the houses will be observed.
\end{abstract}

Key words: corrosion, carbonation, housing, environmental loads.

Recibido: 04 de junio del 2008 • Aprobado: 03 de diciembre del 2008

\section{INTRODUCCIÓN}

Los fenómenos meteorológicos extraordinarios producen grandes e impresionantes daños en las construcciones ubicadas en las costas; sin embargo la mayoría de los deterioros que se observan en las construcciones de concreto reforzado ubicadas en esas zonas se derivan de su exposición cotidiana al medio ambiente; lo anterior puede provocar tanto daños al concreto, como reacciones químicas en el acero de refuerzo, las cuales generalmente producen la ruptura del concreto ubicado en su cercanía. 
Cuando este deterioro se produce en viviendas financiadas con créditos de interés social, las cuales generalmente pertenecen a un estrato de la población con recursos económicos escasos, las acciones de mantenimiento o reparación suelen ocupar un lugar de baja prioridad; de ahí que sus propietarios se limiten a observar como se reduce el valor de sus viviendas y el confort que éstas les proporcionan.

Se ha definido la durabilidad como la propiedad que presenta un material o estructura para resistir la acción del tiempo, ataque químico, abrasión o cualquier otro proceso de deterioro, manteniendo su forma, calidad y condiciones de servicio originales al exponerse a su medio ambiente (Hernández, 2006). Las condiciones de exposición que acarrean los daños más comunes en el concreto provienen de los líquidos y de los gases que lo penetran, tales como dióxido de carbono, agua, oxígeno, cloruros, sulfatos, etc.; estos elementos o compuestos originan diversas reacciones químicas, cuyo efecto más crítico suele ser la corrosión del acero de refuerzo (Lamond, 1997).

La corrosión se define como la interacción de un metal con el medio que lo rodea, produciendo cambios en sus propiedades tanto físicas como químicas. Su característica fundamental es que, generalmente, sólo ocurre en presencia de un electrolito, ocasionando regiones plenamente identificadas, llamadas anódicas y catódicas. En las primeras se da una reacción de oxidación por medio de la cual los electrones son liberados dirigiéndose a las regiones catódicas, produciéndose en el ánodo la disolución del metal.

Entre los agentes de medio ambiente que inducen la corrosión del acero de refuerzo, el dióxido de carbono $\left(\mathrm{CO}_{2}\right)$ es el compuesto que con mayor frecuencia entra en contacto con las construcciones, ya que es un componente natural de la atmósfera. La concentración promedio de $\mathrm{CO}_{2}$ en la atmósfera es de aproximadamente un $0,03 \%$, sin embargo, esta pequeña concentración reacciona lentamente con los hidróxidos de la pasta de cemento que se encuentran diluidos en la solución del poro del concreto, primero con los hidróxidos de sodio y potasio; y posteriormente, los carbonatos que estas reacciones han generados reaccionan con el hidróxido de calcio, dando lugar como producto de esta reacción a la formación de carbonato de calcio que precipita en la superficie del poro. Se dice entonces que esa porción del concreto se ha carbonatado.

Estas reacciones dan como resultado la reducción del $\mathrm{pH}$ de la solución del poro de concreto a un valor tal que la capa de óxidos que protege el acero pierde su estabilidad. Al destruirse esta capa, la corrosión puede desarrollarse con el concurso de oxígeno y humedad.

Otro agente que propicia la destrucción de la capa pasivante del acero son los iones cloruros; el ambiente marino contiene diversas sales las cuales se encuentran disueltas en el agua de mar. Más del $55 \%$ de los solutos sólidos del agua de mar son iones cloruro $\left(\mathrm{Cl}^{-}\right)$, principalmente cloruro sódico, y en menores cantidades cloruro magnésico, cloruro potásico y cloruro cálcico.

También, suelen introducirse al concreto, cloruros contenidos en algunos de sus componentes, como son el cemento y los aditivos acelerantes; o bien por la contaminación involuntaria, y por lo general ignorada, del agua y los agregados.

Los cloruros íntimamente asociados al cemento hidratado no son solubles en agua, por lo que no causan corrosión; sin embargo, algunas investigaciones han mostrado que cloruros que se encuentran unidos a algún compuesto de hidratación del cemento pudieran romper ese enlace y regresar otra vez a la solución de poro (ACI 222, 2001).

La velocidad del ataque por cloruros varía según la región geográfica de acuerdo a la salinidad o a la concentración de las sales contenidas en el agua de mar. Ésta depende principalmente de tres factores: la evaporación, la precipitación pluvial y la proximidad a las descargas de los ríos. El rango de salinidad en los diferentes mares en el mundo va aproximadamente de treinta a cuarenta gramos de sal por litro de solución (Lewis, 1980). La salinidad en las costas de Península de Yucatán se encuentra en el rango entre 35,5 g/l y 36,5 g/l.

Por otra parte, los sulfatos han sido identificados como compuestos que generan deterioro directamente al concreto. Las principales fuentes de sulfatos que pueden atacar al concreto son: los suelos que contienen yeso mineral (sulfato cálcico), las aguas que 
contienen residuos industriales $\mathrm{y}$, principalmente el agua de mar que contiene una concentración de aproximadamente 0,004 de sulfato de sodio.

Cuando el concreto ha endurecido, los iones sulfato que ingresan del ambiente externo reaccionan con los monosulfoaluminatos formando la sal llamada etringita. Esta sal provoca un aumento en volumen del orden del $250 \%$, lo cual produce grandes tensiones que desencadenan fisuras y desprendimientos superficiales del material (Sanjuán y Castro, 2001).

En la Península de Yucatán las viviendas se han construido por muchos años con muros de mampostería y losa de concreto. Los muros inicialmente de mampostería de piedra fueron sustituidos a partir de la década de los sesentas del siglo pasado por muros de mampostería de bloques de concreto, confinada con cadenas (elementos horizontales) y castillos (elementos verticales) de concreto reforzado. En las últimas dos décadas, buscando procesos más rápidos y económicos se ha iniciado la sustitución de éstos, por muros monolíticos de concreto reforzado.

La gran resistencia del concreto ante cargas de compresión y el hecho de que el sistema estructural en cajón sea muy rígido e hiperestático hacen que estructuralmente la combinación de muros y losas de concreto reforzado sea una solución muy eficaz. Sin embargo, regiones en las que el medio ambiente es agresivo con el concreto reforzado, el sistema podría ser vulnerable ante las cargas ambientales y como consecuencia poco durable (Solís et al, 2005). Actualmente se están realizando grandes unidades habitacionales, en el sureste de México, utilizando muros de concreto reforzado.

El presente artículo presenta la evaluación por durabilidad de una muestra de viviendas ubicadas en un ambiente agresivo. El objetivo del estudio fue determinar el impacto que las cargas del ambiente han provocado en los muros de concreto reforzado de las viviendas estudiadas.

\section{METODOLOGÍA}

La unidad habitacional a estudiar se eligió buscando tres características: ubicación en una población costera, construcción con muros de concreto reforzado y edad mínima de diez años. Con lo anterior se buscó que las viviendas cumplieran con las condiciones necesarias para evaluar los efectos de las cargas ambientales severas en el concreto reforzado. Después de definir el sitio de estudio, se obtuvieron de la base de datos del Servicio Meteorológico Nacional los valores promedio de los principales parámetros climáticos.

Una vez seleccionada la unidad habitacional, se revisaron las características constructivas y se hizo una inspección del estado general que presentaban las viviendas. Se eligió una muestra de viviendas para realizar el estudio buscando que no hubieran sido modificadas ni reparadas, y que sus propietarios permitieran realizar las pruebas y extraer las muestras del concreto necesarias.

Se realizó un diagnóstico de los elementos de concreto de las viviendas elegidas, desde el punto de vista de su durabilidad, por medio de las siguientes pruebas:

\subsection{Medición del recubrimiento del concreto}

En condiciones normales, el concreto que envuelve al acero de refuerzo le confiere a éste una buena protección, tanto física como química. El espesor del recubrimiento del acero de refuerzo es un factor importante en el control del transporte de los agentes agresivos; cuanto más grueso e impermeable es el recubrimiento tanto más largo es el intervalo antes de que los gases y líquidos alcancen la posición del acero.

\subsection{Determinación de la profundidad de carbonatación (RILEM, 1988)}

Se utilizó un método de colorimetría para establecer la profundidad del frente de carbonatación, el cual consiste en el tratamiento de la superficie de concreto acabado de romper, con una solución al $1 \%$ de fenolftaleína en alcohol etílico. La coloración del material es un indicador del valor de su potencial de hidrógeno $(\mathrm{pH})$, desde el color púrpura para los valores originales de la alcalinidad del concreto $(>9,5)$ hasta transparencia para valores de alcalinidad bajos $(<8,0)$. 


\subsection{Medición de los potenciales electroquímicos del acero de refuerzo (ASTM C-876,1999)}

Esta prueba se realizó para determinar la posible actividad corrosiva del acero embebido en el concreto. Se utilizó un electrodo de referencia de media celda de cobre/sulfato de cobre conectado a un multímetro digital de 10 Mohms.

\subsection{Estimación de la velocidad de corrosión (ASTM G-59, 2003)}

Las mediciones de la velocidad de corrosión se realizaron aplicando la técnica electroquímica de resistencia a la polarización, la cual consiste en aplicar al acero de refuerzo una señal eléctrica de pequeña amplitud, obteniéndose la resistencia mediante el cociente entre la variación del potencial y de la corriente.

\subsection{Estimación de dureza superficial del concreto (ASTM C-805, 2002)}

La dureza de la superficie del concreto puede ser correlacionada con su resistencia a la compresión axial, que es el principal indicador de la calidad del material; esta característica se determinó en campo por medio de la prueba del martillo de rebote o esclerómetro. Aun cuando se espera que el concreto carbonatado se vuelva más duro, la importancia de la prueba radica en que es no destructiva y que permite la comparación relativa entre diferentes partes de la estructura o estructuras de la misma edad.

\subsection{Determinación de la concentración de cloruros (ASTM C-1218, 1999)}

Esta prueba se realizó en el laboratorio por medio de la disolución de una muestra de polvo de concreto en un medio acuoso, determinándose la concentración por el método del ión selectivo de cloruros, utilizando un electrodo de plata/ cloruro de plata como referencia.

\subsection{Determinación de la concentración de sulfatos (ASTM C-114, 2007)}

Se realizó en el laboratorio por medio de la disolución de la muestra de polvo de concreto en ácido clorhídrico, seguido de una reacción con cloruro de bario, obteniéndose la cantidad de sulfatos como el residuo producto de la desintegración del reactivo después de la calcinación.

\subsection{Medición de la densidad y la porosidad de concreto (ASTM C-642, 2006)}

Estas características fueron obtenidas en el laboratorio a partir de la masa seca, saturada y sumergida de las muestras extraídas de los elementos de cada vivienda, con la finalidad de tener otros indicadores de la calidad del concreto.

\section{RESULTADOS}

El trabajo de investigación se desarrolló en Chetumal, capital del Estado de Quintana Roo, México; esta ciudad se encuentra ubicada frente la Bahía de Chetumal, la cual es compartida con el vecino país de Belice. Se eligió para el estudio una unidad habitacional con antigüedad de quince años, construida para los trabajadores al servicio del gobierno federal.

Las viviendas de esta unidad habitacional tienen un área construida de $88,13 \mathrm{~m}^{2}$; la cimentación es una losa de concreto de $10 \mathrm{~cm}$ de espesor. La estructura es un sistema de cajón, con muros de concreto ( $f^{\prime} \mathrm{c}=20 \mathrm{MPa}$ ) de $12 \mathrm{~cm} \mathrm{de}$ espesor reforzados con malla electrosoldada de acero de 5,72 $\mathrm{mm}$ (fy = 500 MPa), con separación de $15 \mathrm{~cm}$ en ambos sentidos; y sistema de cubierta con losa nervada de concreto ( $\left.\mathrm{f}^{\prime} \mathrm{c}=20 \mathrm{MPa}\right)$ con barras de 7,93 mm (fy $=420 \mathrm{MPa}$ ) y casetones de poliestireno.

Durante la inspección se pudo observar que algunas de las viviendas de la unidad habitacional muestran daños en su estructura, tanto en los muros, como en los nervios de la cubierta. En la Figura 1 se muestran 
desprendimientos del recubrimiento de concreto producto de la corrosión de acero de refuerzo en muro. En la Figura 2 se presentan los daños producidos por causas similares en el nervio perimetral de la cubierta.

Se seleccionaron cinco viviendas las cuales permanecían sin haber sido intervenidas o modificadas respecto a la construcción original. Las pruebas en el concreto y el acero se realizaron en los muros a una altura de 1,5 m con respecto al nivel superior de la cimentación; la extracción de muestras de concreto se realizó a la misma altura. Los resultados de las pruebas de campo realizadas se presentan en el Cuadro 1.

Se midió directamente en los muros la profundidad de la carbonatación del concreto y el recubrimiento del acero; también se

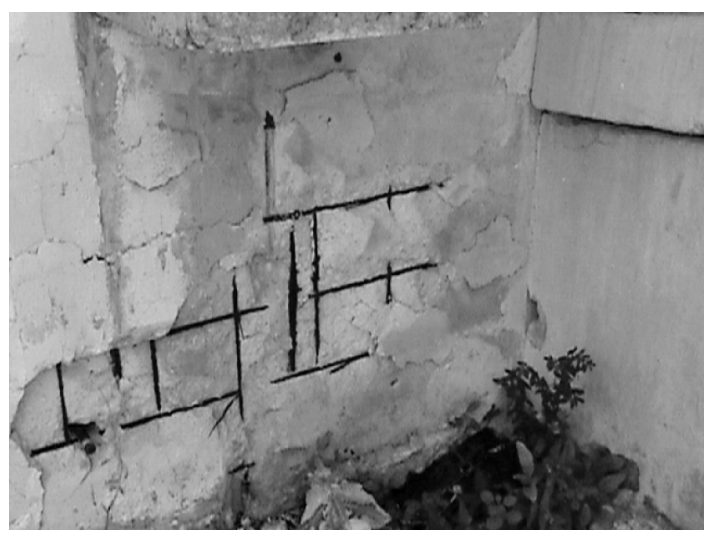

Figura 1. Muro de concreto dañado por corrosión del acero de refuerzo.

Fuente: (Los autores).

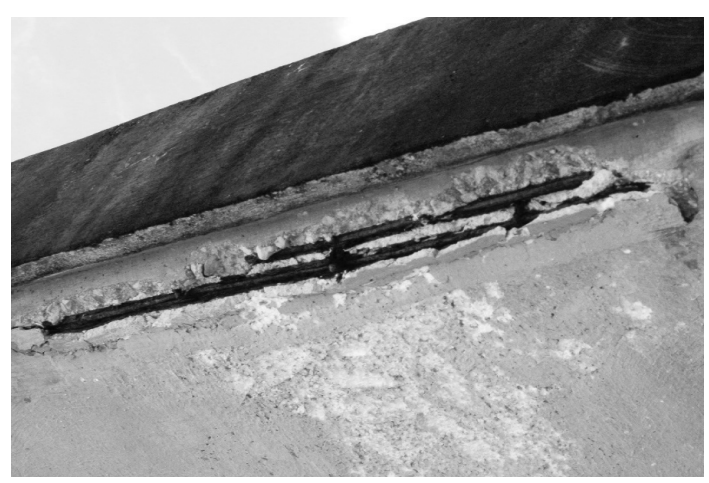

Figura 2. Daños por corrosión en nervio perimetral de la cubierta.

Fuente: (Los autores). estimó en la superficie de los mismos la dureza del concreto, la cual se correlacionó con la resistencia a la compresión del material. En la malla de acero de refuerzo se midieron el potencial, la resistencia eléctrica y la velocidad de corrosión. En la vivienda 5 no fue posible obtener las medidas de la velocidad de corrosión y de la resistencia eléctrica debido a que el sensor de medición no pudo confinar la señal eléctrica

Para obtener las características del concreto, en cada vivienda se extrajeron muestras inalteradas del material. Se midieron en los especímenes extraídos: la densidad, la porosidad y la absorción del concreto. Los resultados de las pruebas se presentan en el Cuadro 2.

Para obtener en el laboratorio las concentraciones de los cloruros y los sulfatos en el concreto, se extrajeron muestras de polvos del material. Los valores graficados de las concentraciones de los cloruros y sulfatos para diferentes profundidades se presentan en la Figuras 3 y 4.

El clima de la región del estudio es cálido subhúmedo y corresponde al tipo que prevalece en la gran mayoría del territorio de la Península de Yucatán. Se caracteriza por tener poco cambio de temperatura entre las diferentes estaciones del año y una estación lluviosa en verano. En el Cuadro 3 se presentan las características climáticas de la ciudad de Chetumal, tomada de la información publicada por el Servicio Meteorológico Nacional (CONAGUA, 2008).

\section{DISCUSIÓN}

El recubrimiento medido a la malla de acero fue adecuado de acuerdo a las prácticas de diseño y construcción recomendadas (ACI 318-05); en la cara de los muros expuesta al medio ambiente el promedio del recubrimiento fue de $64 \mathrm{~mm}$.

Se observó que el frente de carbonatación no ha alcanzado al acero de refuerzo, con una excepción (vivienda 5); por lo que parece que la acción del $\mathrm{CO}_{2}$ no ha sido un factor que ya haya provocado la despasivasión del acero 
Cuadro 1. Resultados de las pruebas de campo.

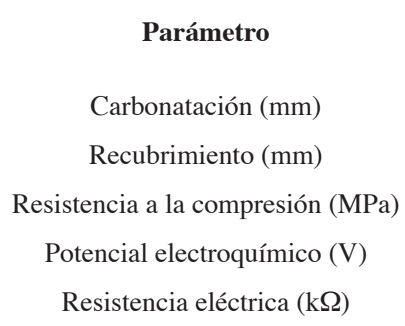

Velocidad de corrosión $\left(\mu \mathrm{A} / \mathrm{cm}^{2}\right)$

$\begin{array}{cc}\mathbf{1} & \mathbf{2} \\ 36 & ---- \\ 60 & 76 \\ 15 & 13 \\ -0,250 & -0,216 \\ 16 & 18 \\ 0,031 & 0,043\end{array}$

Fuente: (Los autores).
Viviendas

$\begin{array}{ccc}\mathbf{3} & \mathbf{4} & \mathbf{5} \\ 55 & 45 & 60 \\ 74 & 56 & 54 \\ 12 & 10 & 13 \\ -0,386 & -0,333 & -0,515 \\ 27 & 1 & ---- \\ 0,020 & 1,154 & ----\end{array}$

Cuadro 2. Resultados de las pruebas de laboratorio realizadas con las muestras de concreto extraídas de los muros.

Parámetro
Densidad $\left(\mathrm{g} / \mathrm{cm}^{3}\right)$
Porosidad $(\%)$
Absorción $(\%)$

\begin{tabular}{ccccc}
\multicolumn{5}{c}{ Viviendas } \\
$\mathbf{1}$ & $\mathbf{2}$ & $\mathbf{3}$ & $\mathbf{4}$ & $\mathbf{5}$ \\
1,929 & 1,956 & 2,037 & 2,131 & 2,221 \\
26 & 36 & 29 & 29 & 28 \\
17,5 & 24,4 & 17,6 & 16,7 & 15,4 \\
& Fuente: (Los autores). & & &
\end{tabular}

Cuadro 3. Caracterización del clima de Chetumal.

\author{
Temperatura máxima \\ promedio
}

$\left({ }^{\circ} \mathrm{C}\right)$

31

\author{
Temperatura mínima \\ promedio
}

$\left({ }^{\circ} \mathrm{C}\right)$

22
Precipitación anual

(mm)

1,327
Evaporación anual

(mm)

1,803

Fuente: (CONAGUA, 2008).

en la mayoría de las viviendas. Utilizando un modelo simplificado (Ecuación 1) para modelar la velocidad del avance del frente de carbonatación se determinó un coeficiente de carbonatación $(k)$ con un valor promedio de 12,7 $\mathrm{mm} / \mathrm{año}{ }^{1 / 2}$.

$X=k \sqrt{t}$

La Figura 5 presenta la gráfica que se obtuvo de la ecuación (1) con el coeficiente promedio calculado. Si se considera el valor del recubrimiento promedio medido, se esperaría que a la edad de 25 años el frente de carbonatación ya haya alcanzado por completo la malla de refuerzo de todas las viviendas.

En una investigación previa, Solís et al (2007) obtuvieron coeficientes de carbonatación para concretos de diferentes relaciones A/C (de 0,4 a 0,7 ) expuestos a la carbonatación natural en el mismo clima; extrapolando sus resultados el coeficiente promedio calculado en las viviendas correspondería a una relación $\mathrm{A} / \mathrm{C}$ de 0,85 , el cual a su vez, es característico de un concreto de aproximadamente $18 \mathrm{MPa}$ de resistencia a la compresión (Solís et a., 2008).

La resistencia a la compresión del concreto estimada a partir de la prueba no destructiva 


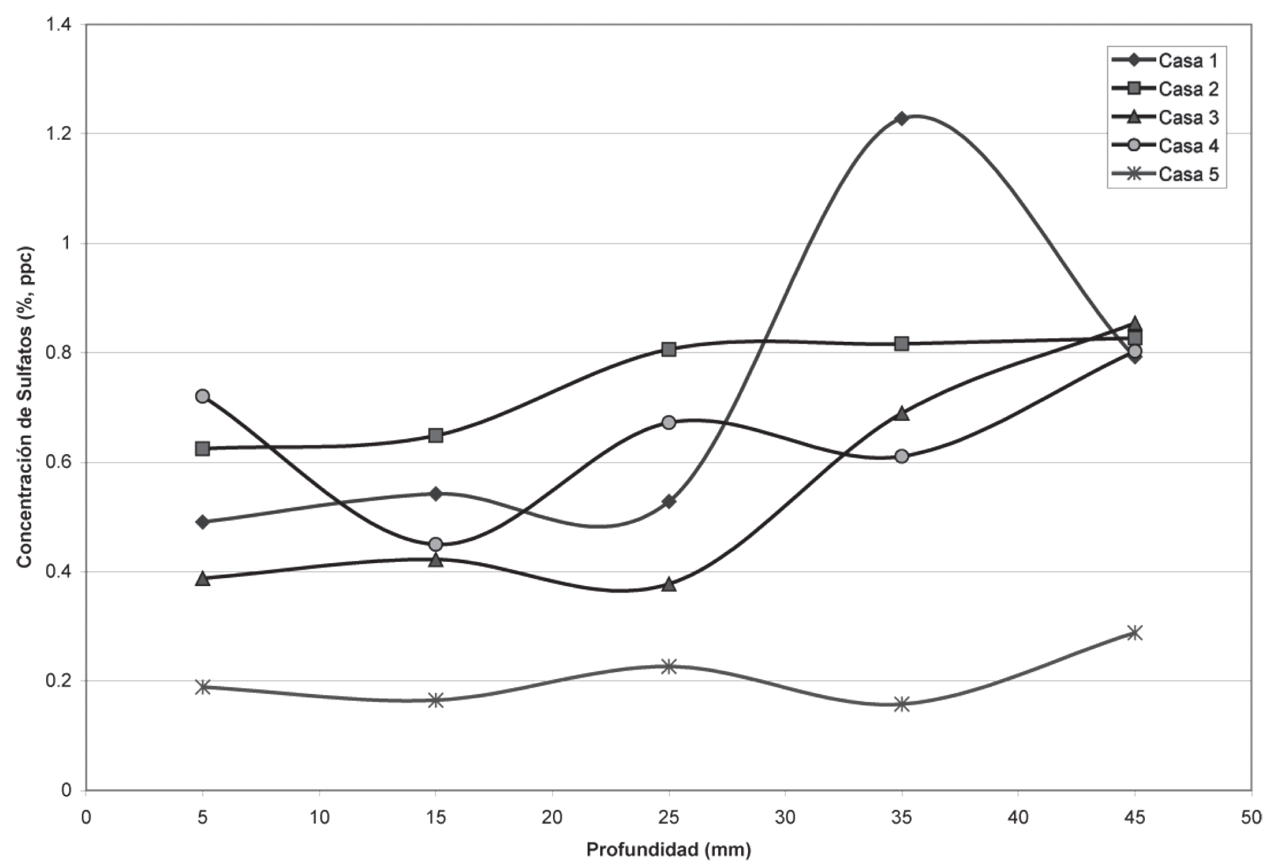

Figura 4. Concentración de sulfatos por vivienda (\% por masa de concreto).

Fuente: (Los autores).

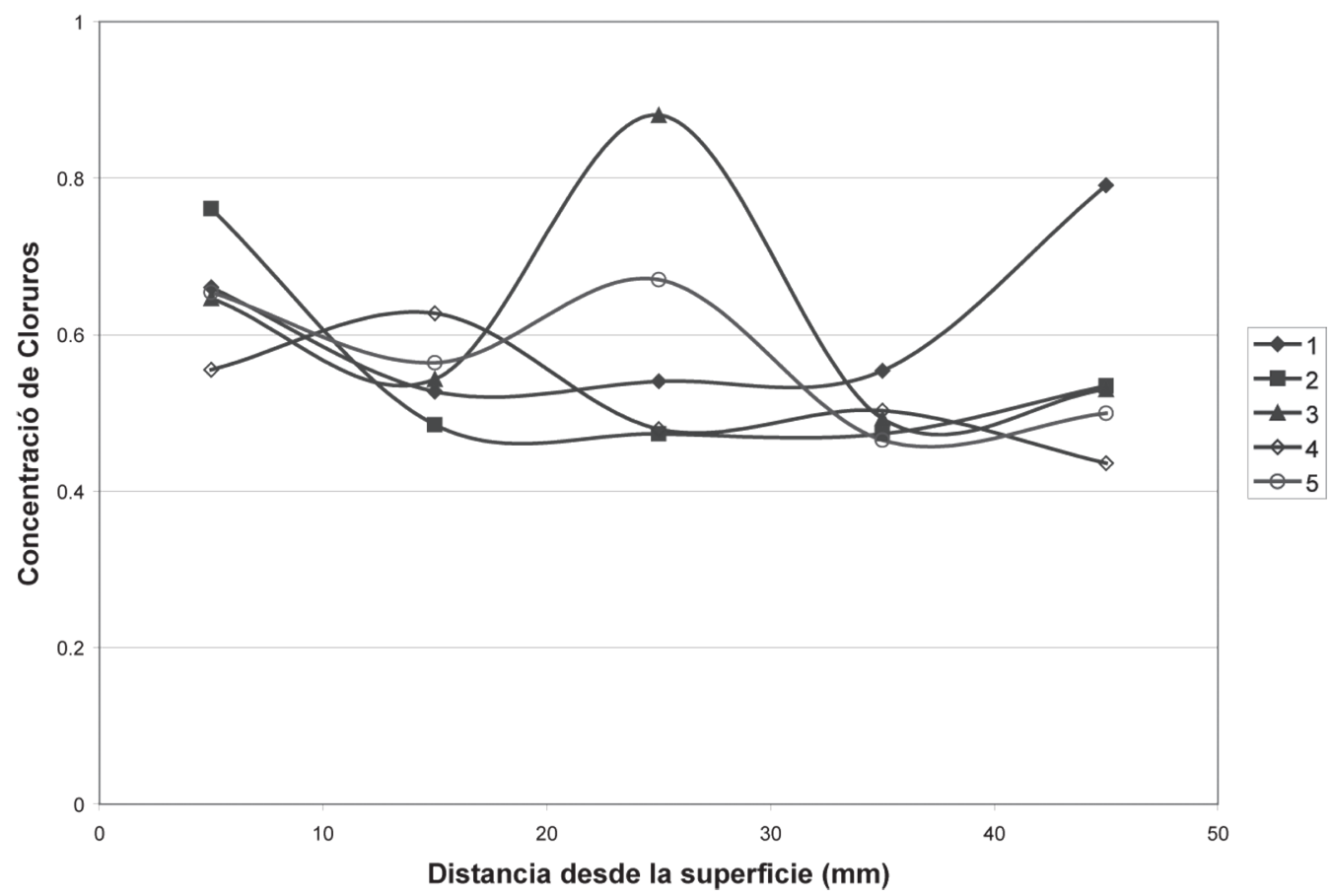

Figura 3. Concentración de cloruros por vivienda $\left(\mathrm{kg} / \mathrm{m}^{3}\right.$ de concreto).

Fuente: (Los autores). 
realizada en los muros resultó muy baja en las cinco viviendas con respecto a la de diseño (20 $\mathrm{MPa}$ ); las valores de la resistencia se estimaron en un rango que abarca el $50 \%$ y el $75 \%$ de la f'c.

Con relación al potencial electroquímico medido en el acero de refuerzo, en dos de las viviendas (3 y 4) fue más negativo que -300 $\mathrm{mV}$, lo cual indicaría, según los criterios de la norma ASTM C 876, una probabilidad de media a alta de estar ocurriendo la corrosión; y en una vivienda (5) el potencial fue menor a $-400 \mathrm{mV}$ lo que indicaría una alta probabilidad de corrosión en el acero.

Las velocidades de corrosión medidas, con excepción de una vivienda (4), estuvieron en un rango que se interpreta como de corrosión despreciable según el Manual de la Red Durar (Troconis-Rincón et al, 2000). Sin embargo, en la vivienda 4 se midió una intensidad comparativamente muy alta $\left(1,154 \mu \mathrm{A} / \mathrm{cm}^{2}\right)$, que se interpreta como una velocidad de corrosión muy elevada.

En general, los potenciales indicaron probable corrosión del acero de refuerzo, mientras que las velocidades de corrosión medidas indicaron valores de pasividad. Es probable que estos valores de pasividad pudieran estar encubiertos por la falta de electrolito en el concreto, ya que las pruebas fueron realizadas en otoño que corresponde a la temporada de seca de la región, y la mayoría de las viviendas mostraron resistencias eléctricas en el orden de $20 \mathrm{k} \Omega$. Este efecto de la humedad se aprecia en los resultados de las dos pruebas en la vivienda 4 , que presentó valores de resistencia eléctrica diez veces menor y velocidad de corrosión cien veces mayor, con relación a las otras viviendas.

Los resultados de la porosidad del concreto fueron superiores al $15 \%$ en todos los casos; de acuerdo al reporte de Andrade (1988), el concreto sería muy permeable e inadecuado para proteger el acero. Los altos valores medidos confirman que la calidad del concreto fue inferior a la requerida en el proyecto. Sin embargo, Solís y Moreno (2006) han reportado porosidades en un rango entre el $16 \%$ y el $23 \%$ para concreto de diferentes calidades preparados con los mismos agregados producto de la trituración de piedra caliza, los cuales son muy absorbentes y porosos, pero que

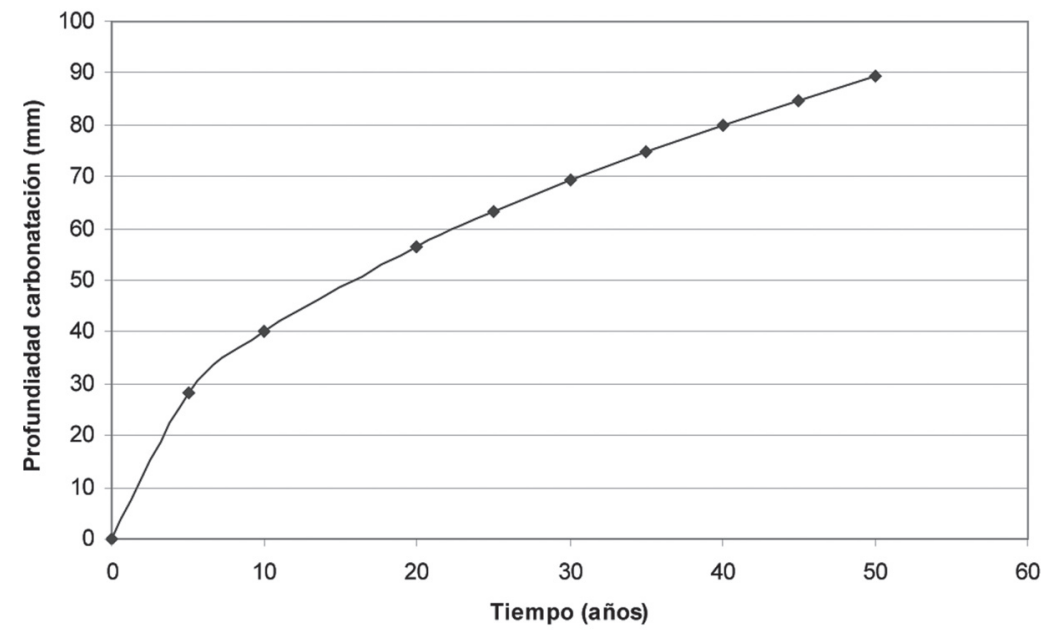

Figura 5. Modelo simple de avance del frente de carbonatación.

Fuente: (Los autores). 
pueden dar lugar a concretos de buena calidad utilizando una relación $\mathrm{A} / \mathrm{C}$ adecuada.

De acuerdo al reporte del ACI 222 (2001), el umbral de cloruros que provoca la despasivasión del acero se encuentra entre $0,6 \mathrm{~kg} / \mathrm{m}^{3}$ y 0,9 $\mathrm{kg} / \mathrm{m}^{3}$. En todas las viviendas la concentración de cloruros rebasa el valor de 0,6 en alguna profundidad; sinembargo,únicamentelavivienda 1 rebasa esta concentración a una profundidad cercana al acero. Estudios realizados en la Península de Yucatán, con concretos preparados con el mismo tipo de agregado, han reportado niveles iniciadores de la corrosión entre $(1,0 \mathrm{y}$ 2,0) $\mathrm{kg} \mathrm{de} \mathrm{Cl}^{-}$por $\mathrm{m}^{3}$ de concreto. De ahí que, en lo general, parecería que los iones cloruros no han despasivado el acero aún a la edad del estudio (Castro, 2001).

Para comparar la concentración de sulfatos encontrados en el concreto, se midió la concentración original de sulfatos en concretos preparados en laboratorio con tres $\mathrm{A} / \mathrm{C}$ y $\sin$ exposición alguna al medio ambiente agresivo; los resultados promedios fueron: un $0,9 \%$ en masa de concreto, para $\mathrm{A} / \mathrm{C}$ de 0,5 ; un $0,6 \%$ para $\mathrm{A} / \mathrm{C}$ de 0,6 ; y un $0,8 \%$ para $\mathrm{A} / \mathrm{C}$ de 0,7 . Los valores máximos medidos en las viviendas estuvieron alrededor del $0,8 \%$, con excepción de una vivienda (1) que rebasó el 1,2\%; de ahí que se pueda interpretar que la penetración de sulfatos debido a los quince años de exposición en el medio ambiente ha sido despreciable, tomando en cuenta la concentración de sulfatos obtenidos en los concretos de control no sujetos a exposición alguna.

\section{CONCLUSIONES}

El concreto resultó de calidad inferior a la especificada y a la requerida para que sea suficientemente impermeable y garantice la durabilidad de las viviendas.

Se espera que en pocos años (de cinco a diez) el frente de carbonatación haya alcanzado al acero de refuerzo de los muros produciendo la despasivasión generalizada de la malla de refuerzo.

En conjunto, las mediciones electroquímicas sugieren una probabilidad media de que esté ocurriendo la corrosión actualmente.
Las concentraciones de cloruros halladas indican que el acero aun no habría sido despasivado por esta acción; sin embargo a profundidades menores ya han penetrado suficientes iones para provocar en el futuro la despasivasión.

No se encontraron concentraciones de sulfatos superiores a las que contiene el concreto en forma natural producto de sus reacciones de hidratación.

Se esperan daños generalizados en los muros a una edad de veinticinco años por el efecto sinérgico de la pérdida de alcalinidad del concreto y el avance de los iones cloruros.

\section{RECONOCIMIENTOS}

Los autores agradecen al FOMIX CONACYTGobierno de Estado de Quintana Roo (Proyecto QROO-C03-04-001), a la Universidad Autónoma de Yucatán y al CINVESTAV por el apoyo para realizar la investigación. Así como a P. Castro, M. Balancán, A. Nadal, F. Cauich y R. Pat su colaboración en la ejecución de algunas de las pruebas reportadas.

\section{REFERENCIAS BIBLIOGRÁFICAS}

ACI-222 (2001). Protection of metals in concrete against corrosion, American Concrete Institute, Farmington Hills, MI.

ACI-318 (2005). Building code requirements for structural concrete and commentary, American Concrete Institute, Farmington Hills, MI.

Andrade, C. (1988). Manual Inspección de obras dañadas por corrosión de armaduras, Instituto Eduardo Torroja de Ciencias de la Construcción, Madrid.

ASTM C-114. (2007). Standard test methods for chemical analysis of hydraulic cement, ASTM Book of Standards Volume 04.02: Construction: Concrete and Aggregates, ASTM International, West Conshohocken, PA. 
ASTM C-642. (2006). Standard test method for density, absorption, and voids in hardened concrete, ASTM Book of Standards Volume 04.02: Construction: Concrete and Aggregates, ASTM International, West Conshohocken, PA.

ASTM C-805. (2002). Standard test method for rebound number of hardened concrete. ASTM Book of Standards Volume 04.02: Construction: Concrete and Aggregates, ASTM International, West Conshohocken, PA.

ASTM C-876. (1999). Standard test method for half-cell potentials of uncoated reinforcing steel in concrete, ASTM Book of Standards Volume 04.02: Construction: Concrete and Aggregates, ASTM International, West Conshohocken, PA.

ASTM C-1218. (1999). Standard test method for water-soluble chloride in mortar and concrete. ASTM Book of Standards Volume 04.02: Construction: Concrete and Aggregates, ASTM International, West Conshohocken, PA.

ASTM G-59. (2003). Standard test method for conducting potentiodynamic polarization resistance measurements, ASTM Book of Standards Volume 03.02: Wear and Erosion; Metal Corrosion, ASTM International, West Conshohocken, PA.

Castro, P. (2001). Corrosión en estructuras de concreto armado: teoría, inspección, diagnóstico, vida útil y reparaciones, $2^{\mathrm{a}}$ ed., IMCYC, México.

CONAGUA Servicio Meteorológico Nacional, recuperado el 10 de enero de 2008 en: http:// smn.cna.gob.mx/

Hernández, O. \& Mendoza, C. J. (2006). Durabilidad e infraestructura: retos e impacto socioeconómico, Ingeniería Investigación y Tecnología, UNAM, 7(1), 57-70.
Lamond, J. F. (1997, Nov). Designing for durability, Concrete International. 34-36.

Lewis, E. L. (1980). Escala práctica de salinidad 1978 y sus antecedents, IEEE J. Ocean. Eng., OE-5(1): 3-8.

RILEM. (1988). Recommendation, measurement of hardened concrete carbonation depth, Materials and Structures 21, 453.

Sanjuán, M. \& Castro, P. (2001). Acción de los agentes químicos y físicos sobre el concreto, México: IMCYC.

Solís, R. \& Moreno, E. (2006). Análisis de la porosidad del concreto con agregado calizo, Revista de la Facultad de Ingeniería de la Universidad Central de Venezuela, 21(3), 101-109.

Solís, R., Moreno, E. \& Alcudia, C. (2008). Estudio de la resistencia del concreto por el efecto combinado de la relación aguacemento, la relación grava-arena y el origen de los agregados, Revista Técnica de la Universidad de Zulia, 31(3), 1-12.

Solís, R., Moreno, E. \& Castro, P. (2005). Durabilidad en la estructura de concreto de vivienda en zona costera, Ingeniería: Revista Académica de la Facultad de Ingeniería Universidad Autónoma de Yucatán, 9(1), 13-18.

Solís, R., Moreno, E. \& Estrella C. (2007). Carbonatación natural del concreto con agregado calizo, En: IX Congreso Latinoamericano de Patología y XI Congreso de Control de Calidad en la Construcción, Quito, Ecuador.

Troconis-Rincón, O., Romero-Carruyo, A., Andrade, C., Helene, P. \& Díaz I., (2000). Manual for inspecting, evaluating and diagnosing corrosion in reinforced concrete structures. Durar Network Maracaibo: CYTED. 


\section{SOBRE LOS AUTORES}

\section{Rómel Solís Carcaño}

Ingeniero Civil, Maestro en Ingeniería

Investigador y profesor de la Facultad de Ingeniería de la Universidad Autónoma de Yucatán, México.

Teléfono: 52-999-930-0568

Apartado postal: 150 Condomex, Mérida, México

Facsímil: 52-999-930-0559

Correo electrónico: tulich@uady.mx

\section{Eric Iván Moreno}

Ingeniero Civil, Doctor en Ingeniería

Investigador y profesor de la Facultad de Ingeniería de la Universidad Autónoma de Yucatán, México. Teléfono: 52-999-930-0568

Apartado postal: 150 Condomex, Mérida, México

Facsímil: 52-999-930-0559

Correo electrónico: emoreno@uady.mx

\section{Luis Felipe Jiménez Torrez}

Ingeniero Civil, Maestro en Ingeniería-Construcción Profesor de la Facultad de Ingeniería Civil, del Instituto Tecnológico de Chetumal, Departamento de Ciencias de la Tierra.

Teléfono: 52-9838-321019 o 9838-370603

Apartado postal:

Facsímil: 52-9838-322330

Correo electrónico: fjtorrez@prodigy.net.mx 
